\title{
Vivir con miedo: un estudio sobre las agendas digitales y las fuentes de información del delito, la violencia y el riesgo
}

\author{
Esteban Zunino \\ CONICET, Universidad Nacional de Cuyo. \\ estebanzunino@hotmail.com \\ Fecha de finalización del trabajo: 25 de abril de 2020 \\ Recibido: 6 de agosto de 2020 . \\ Aceptado: 16 de octubre de 2020. \\ DOI: https://doi.org/10.26422/aucom.2020.0902.zun
}

\section{Resumen}

El presente artículo tiene por objetivo analizar las agendas informativas sobre delitos, violencias y riesgos construidas por los principales medios digitales de Argentina: Clarín, La Nación e Infobae durante 2019. A partir de un análisis de contenido cuantitativo, se pretende establecer qué porcentaje del hueco informativo de las portadas digitales ocupan este tipo de asuntos y cuáles son los actores que logran instituirse como fuentes confiables de información sobre los principales tópicos que conforman la agenda del miedo y del temor. El trabajo empírico se desarrolló a partir de la operacionalización de conceptos provenientes de las teorías de la agenda setting, el indexing y la hipótesis del standing.

Palabras clave: delito, violencia, riesgo, medios, agenda, fuentes.

\section{Living in fear: the online news agenda and sources of information on crime, violence, and risk}

\section{Abstract}

This paper analyzes the news agenda - in regard to crime, violence, and risk - as set during 2019 by the main online news sites in Argentina: Clarín, La Nación, and Infobae. Through a quantitative content analysis, we determine how much online real estate these topics occupy on the home screens of the above news sites. In addition, we identify the social actors who emerge as reliable sources of information on matters pertaining to this public fear agenda. Our empirical work puts into practice the theories of "agenda setting "and "indexing", and the hypothesis of media "standing".

Keywords: crime, violence, fear, media, agenda, information sources. 


\title{
Vivendo com o medo: um estudo sobre agendas digitais e fontes de
} notícias sobre crime, violência e risco

\begin{abstract}
Resumo
O objetivo deste artigo é analisar as agendas informativas sobre crimes, violência e riscos construídas pelas principais mídias digitais da Argentina: Clarín, La Nación e Infobae. A partir de uma análise de conteúdo quantitativa, pretende-se estabelecer qual é o percentual da lacuna informacional das capas digitais ocupada por esse tipo de tema e quais são os atores que conseguem se estabelecer como fontes confiáveis de informação sobre os principais temas que compõem a agenda do medo e do crime. O trabalho empírico foi desenvolvido a partir da operacionalização de conceitos das teorias da agenda setting, indexing e hipótese do standing.
\end{abstract}

Palavras chave: crime, violência, risco, mídia, agenda, fonte.

\section{Introducción}

La criminalidad y la violencia ocupan un lugar central en las agendas política, mediática y pública de América Latina (Lagos y Dammert, 2012). En buena medida, eso se explica porque el continente contiene a 18 de los 20 países más violentos del planeta. Si bien el Cono Sur (Argentina, Chile y Uruguay) ostenta tasas de homicidios y victimización más cercanas al promedio mundial (6,2 cada 100 mil habitantes), muchas de las naciones del Caribe -como Honduras, El Salvador y Guatemala, a las que se les suman Venezuela, México y Brasil- exhiben tasas superiores a 20 homicidios por cada 100 mil habitantes (Anitua, 2010), cuando valores superiores a 10 son considerados epidémicos por la Organización Mundial de la Salud (Ávila, 2016).

En la Argentina, las tasas de delito se incrementaron dos veces y media desde la década del 80 , y si bien los homicidios alcanzan a 5,5 por cada 100 mil habitantes (Sozzo, 2016), la "inseguridad" constituye una preocupación central de los ciudadanos. La discusión securitaria cobró un nuevo impulso desde la asunción de Mauricio Macri a la presidencia en 2015. En 2016, el Poder Ejecutivo Nacional manifestó su intención de incrementar medidas punitivistas para combatir tanto el delito organizado como el amateur. Algunas de ellas marcaron continuidades con respecto al Gobierno anterior, como el envío de fuerzas federales a "zonas calientes" (Rodríguez Alzueta, 2014), como la provincia de Buenos Aires y Santa Fe. Otras, asentadas en viejos conceptos promovidos por diversos sectores políticos, condensaron en un proyecto para bajar la edad de imputabilidad de 16 a 14 años. También se adoptaron medidas concretas referidas al control de migraciones, como el Programa de Información Anticipada de Pasajeros (API), por el que se le solicita a las aerolíneas informes sobre los extranjeros que trasladan, y el Decreto 70/2017, ${ }^{1}$ que modifica la Ley 25871 de Migraciones y promueve trámites abreviados de expulsión para extranjeros condenados por haber delinquido

Disponible en https://www.boletinoficial.gob.ar/\#!DetalleNorma/158336/20170130. 
en el país o en el exterior, la denegatoria o retiro de residencia permanente y el impedimento de ingreso o reingreso por diferentes lapsos de tiempo. Finalmente, el Gobierno informó en 2018 su decisión de que las Fuerzas Armadas intervinieran en la seguridad interior en la denominada "lucha contra el narcotráfico", aun en contra de lo que indica la legislación vigente.

Sin embargo, la criminalidad que habitualmente se reconoce como tal remite solo a una porción de los delitos que ocurren en nuestras sociedades. En ese sentido, los ilícitos que los medios suelen englobar dentro de la noción de "inseguridad", 2 es decir, aquellos que se dan contra la propiedad (hurtos y robos concretados o no), contra las personas (lesiones y otras agresiones dolosas) y homicidios (Kessler, 2014) dominan las agendas mediática, política y pública de América Latina en general y de la Argentina en particular (Kessler, 2014; Sozzo, 2016). Desde comienzos del siglo XXI hubo en la Argentina un incremento de la representación mediática del delito (Calzado, 2012; Fernández Pedemonte, 2010) que coincide con el aumento del sentimiento de inseguridad, es decir, la sensación de indefensión de los individuos contra una amenaza aleatoria que opera con autonomía relativa respecto a los hechos delictivos (Kessler, 2009).

Asimismo, los delitos, las violencias y los riesgos representados en las noticias exceden a los sucesos que habitualmente se engloban dentro de la noción de "inseguridad". Ilícitos económicos, laborales, ambientales, pánicos morales y ciertas contravenciones urbanas se despliegan en los medios, construyendo un medioambiente amenazante.

Este trabajo analiza las representaciones mediáticas de diferentes tipos de delitos, violencias y riesgos, partiendo de la premisa de que por tratarse de temas mayormente no experienciales (Zucker, 1978), los medios podrían desempeñar allí un rol más potente en la construcción de imaginarios sociales que con respecto a otro tipo de asuntos más ligados a la experiencia de los sujetos. En términos específicos, se pretende establecer qué lugar ocupan las noticias sobre los diferentes tipos de delitos, violencias y riesgos en las agendas de los principales medios digitales de la Argentina, qué actores intervienen en ellas y cuáles son las fuentes más exitosas a la hora de transmitir sus puntos de vista.

\section{Agendas, delito y medios}

Existe consenso acerca de que los medios pueden, bajo determinadas condiciones, desempeñar un rol significativo en la creación de un estado de riesgo que suele traer aparejadas demandas de mayor control social y punitividad (Entel, 2007; Focas, 2014; Kessler y Martini, 2012). Las piezas periodísticas sobre el crimen constituyen un lugar

Kessler (2009) sostiene que inseguridad es una "prenoción sociológica", es decir, "una forma de explicar la realidad del sentido común antes que un concepto desarrollado por las ciencias sociales" (p. 11). 
Esteban Zunino

Vivir con miedo: un estudio sobre las agendas digitales y las fuentes de información del delito, la violencia y el riesgo

de entrecruzamiento entre la realidad y la ficción que recae habitualmente en el sensacionalismo. Operan en ellas ciertos sesgos, como el de personalización y descontextualización (Bennett, 1991), mediante los cuales los hechos son presentados de manera aislada y sus causales son atribuidas mucho más a las motivaciones individuales de quienes delinquen que a los complejos determinantes estructurales que los condicionan. En tanto, las fuentes consultadas por los periodistas se reducen casi únicamente a las oficiales, especialmente la policía, funcionarios judiciales y del Poder Ejecutivo, lo que contribuye a la descontextualización y parcialidad en la interpretación de los hechos (Martini, 2012).

La mediatización del delito genera lo que Fernández Pedemonte (2010, p. 74) denomina "olas de violencia". Los casos conmocionantes cubiertos por la prensa modifican los criterios de selección de las redacciones. La construcción de series, en las que el tratamiento binario entre lo legal e ilegal se reproduce y normaliza, genera la conexión y consecuente amplificación de cierto tipo de hechos no necesariamente ligados entre sí. Por lo tanto, ciertos casos dramáticos constituyen lo que Brosius y Eps (1995, p. 393) denominan "eventos clave", es decir, prototipos de cobertura que poseen esquemas informativos establecidos y funcionan como estereotipos, provocando que los hechos similares tengan más probabilidad de ser seleccionados.

El impacto de estos eventos en las rutinas productivas condiciona las agendas informativas. Estas son concebidas como un listado de temas y atributos que los medios proponen al público con potencial impacto en las percepciones de las audiencias (McCombs y Valenzuela, 2014). El otorgamiento de relevancia a noticias sobre delitos, violencias y riesgos en la superficie mediática, dada la lejanía de este tipo de asuntos con la experiencia directa de la mayoría de los sujetos (Zucker, 1978), incrementaría la potencial influencia de esas agendas sobre la opinión pública (McCombs, 2006).

Pero los procesos de selección, omisión y jerarquización que se dan en el seno de las redacciones no se restringen a los temas. Los actores portadores de información de primera mano -las fuentes de información- también resultan centrales para la fisonomía de la noticia (Koziner, 2018a).

Según la teoría del indexing (Bennett, 1990), la estructuración de temas y fuentes suele ser elaborada en función de la visión dominante de los Gobiernos acerca de un asunto determinado, producto del poder que poseen las fuentes oficiales, especialmente las estatales (Steele, 1997), con mayor nivel de institucionalización. Entre periodistas y fuentes suele darse una relación simbiótica (Bagdikian, 1985): los medios necesitan de las fuentes para obtener la información y las fuentes dependen de los periodistas para lograr imponer su versión de los hechos. Con ello, según Bennett, Lawrence y Livingston (2007), los medios han cedido el monopolio noticioso a los funcionarios 
públicos, con lo que se restringe la diversidad de voces en detrimento de una mirada amplia y contextualizada de la información.

Sin embargo, Charron (1995) llama la atención sobre una cuestión metodológica clave para el estudio de las fuentes informativas. Según el autor, la medición de la presencia de las fuentes en la superficie de la agenda mediática poco dice de la real influencia de estas en el establecimiento de la agenda, ya que

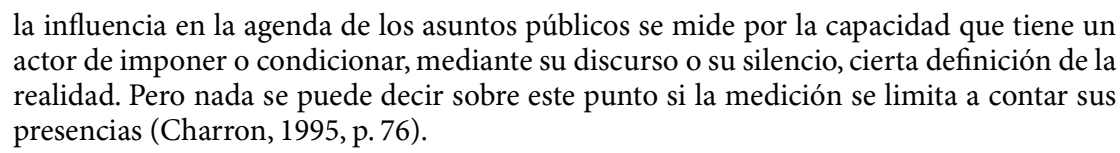

Con el fin de subsanar los defectos en la estimación del peso real de cada fuente en la definición una situación, este trabajo toma noción de standing propuesta por Ferree, Gamson, Gerhards y Rucht (2002). Esta es concebida como el crédito -o descréditoque los medios de comunicación les otorgan a los puntos de vista expresados por las fuentes (Koziner, 2018b). Así, siguiendo a Charron (1995), este estudio no solo tiene en cuenta la visibilidad diferencial de cada fuente, sino también el nivel de crédito que estas obtienen en el tratamiento informativo.

\section{Los medios digitales}

Los medios online cumplieron ya más de dos décadas. Nacidos en Iberoamérica en 1995, la actualidad los encuentra en un momento de reconfiguraciones estructurales. En primer lugar, debido a que la digitalización modificó sustancialmente los modos de consumo de información de las audiencias (Mitchelstein y Boczkowski, 2017). En segundo lugar, porque todo el ecosistema de medios se vio afectado por la irrupción de la sociedad de la información (Scolari, 2014). Finalmente, porque ese proceso de desestructuración, que aún no ha culminado, trastocó los fundamentos básicos de la profesión periodística (Martini y Luchessi, 2004; Retegui, 2017; Salaverría, 2016).

Uno de sus rasgos centrales es la debilidad de sus modelos de negocio (GarcíaAlonso Montoya, 2014). Si en un comienzo la gratuidad fue la norma, entrado el siglo XXI algunos de los grandes diarios online -como El País de España- intentaron financiarse a través de suscripciones. Sin embargo, los resultados no fueron los esperados (Salaverría, 2016). El traspié generó un retorno a la gratuidad que tuvo como contracara una suspensión de la inversión e innovación en medios digitales, también asociada al declive de las empresas que operaban a través de internet a principios de los años 2000. En la actualidad, la tendencia implantada en 2011 por The New York Times consiste en un modelo mixto o de muro de pago "poroso". El modelo es de suscripción y 
Esteban Zunino

Vivir con miedo: un estudio sobre las agendas digitales y las fuentes de información del delito, la violencia y el riesgo

habilita un número acotado de notas gratis, solicitando luego un pago para acceder a la totalidad del home.

Los criterios de noticiabilidad predominantes en el medio digital son la inmediatez y la espectacularización (Martini, 2017), mientras que la noticia es cada vez más "aquello que sucede cerca en el tiempo del público" (Martini, 2017, p. 21). La competencia de los diarios online con otras fuentes de información, como las redes sociales, los ha llevado a un "sensacionalismo argumentativo" que se potencia en las noticias sobre delitos e inseguridad (Martini, 2017, p. 20), instrumentado como una nueva estrategia para atraer la atención de los lectores en un entorno convergente.

En la Argentina, según la Encuesta Nacional de Consumos Culturales 2017, los medios digitales tienen una penetración del 25,6\%. Si bien aún están lejos del 37,4\% de la prensa gráfica (en franco retroceso), se debería considerar que muchos de los que declaran informarse por redes sociales $(23,4 \%)$ terminan accediendo de manera incidental (Mitchelstein y Boczkowski, 2017) a noticias manufacturadas por empresas mediáticas tradicionales que distribuyen sus contenidos a través de múltiples plataformas, lo que aumenta potencialmente el nivel de consumo de medios online a prácticamente la mitad de la población (Sistema de Información Cultural de la Argentina [SINCA], 2017).

En función del andamiaje conceptual precedente se proponen las siguientes hipótesis:

H 1: las noticias vinculadas con diferentes situaciones delictuosas, violentas o presentadas en términos de riesgos para la ciudadanía adquieren niveles importantes de frecuencia informativa en las agendas digitales.

H 2: en el tratamiento mediático del delito y la violencia suelen prevalecer las fuentes estatales, especialmente las fuerzas de seguridad y el Poder Judicial.

\section{Metodología}

La estrategia metodológica está planteada con el propósito de viabilizar la realización de los objetivos planteados. Se desarrolló un análisis de contenido de la cobertura mediática de todas las noticias que incluyeran la comisión de un delito, ilícito o que relataran una situación violenta o de riesgo para la vida de las personas. El estudio se enfocó en las piezas más importantes de los medios digitales, es decir, aquellas que se publicaron en sus portadas o primeros scrolls. Así, se utilizó la técnica de la semana construida (una semana construida por mes) y se analizaron las cinco primeras noticias de los portales de Clarín, La Nación e Infobae entre los meses de abril y octubre de 2019 en dos cortes diarios: a las 9 y 19 horas. Este método permite obtener una muestra aleatoria de la información de los medios ampliando el rango de su selección, 
Austral Comunicación

Volumen 9, número 2 (Diciembre de 2020): 553-582. ISSN 2313-9129

lo que evita distorsiones propias de un momento informativo reducido, al tiempo que suele conservar las marcas de la pauta editorial. La semana se construye partiendo de la edición de un lunes a la que seguirá la edición del martes de la semana siguiente y así con cada día hasta llegar al domingo (Téramo, 2006).

El análisis de contenido permite examinar los mensajes mediáticos, reconstruir su arquitectura, conocer su estructura, componentes básicos y funcionamiento y realizar inferencias reproducibles y válidas sobre fenómenos distintos a los directamente observados (Neuendorf, 2002). Mediante mecanismos lógicos, el analista saca deducciones sobre la porción de la realidad a la que alude la información, así como el contexto en el que esta es producida (Krippendorff, 1990). A partir de esta técnica, se analizaron 1.440 piezas informativas.

Los periódicos, unidades de contexto de la investigación, se seleccionaron por su importancia en términos de circulación, ya que Infobae, Clarín y La Nación se ubican al tope de la preferencia de los lectores de medios digitales de la Argentina, según los datos de la consultora internacional Comscore (Argentina: Infobae reafirma..., 2019).

\section{Unidades de contexto}

Los medios analizados constituyen las unidades de contexto de la investigación. La Nación fue el primer diario de Buenos Aires en entrar en la web, el 1 de diciembre de 1995. De ese modo, uno de los diarios centenarios del país, fundado por el expresidente Bartolomé Mitre (1862-1868) en 1870, empezaba a desempeñarse en la red con un promedio inicial de 1.500 visitas diarias, con un alto porcentaje de lectores que lo hacían desde el exterior (Rost y Bergero, 2016).

El 10 de marzo de 1996 llegó a internet Clarín, el diario en soporte papel más leído del país y uno de los de mayor circulación de habla hispana (Albornoz, 2006). Fundado por el exdiputado del Partido Socialista Independiente Roberto Noble, el 28 de agosto de 1945, es la marca insignia del mayor multimedio argentino (Becerra, 2015). Fue concebido como un medio online pensado para una plataforma digital y no simplemente un volcado de los contenidos del papel. Desde sus inicios, incluyó chats para interactuar con el público, fotos 360 grados, cámaras en vivo, entrevistas online y los noticieros de Radio Mitre, emisora del mismo conglomerado mediático (Rost y Bergero, 2016).

Infobae es el nativo digital más exitoso de la Argentina, ubicándose al tope de las preferencias de medios digitales en el país. Creado en 2002 por el empresario mediático Daniel Hadad, ya vivió siete diseños. Su sello distintivo ha sido el uso del video y, desde 2013, las transmisiones en vivo durante ocho horas desde su estudio de televisión. Según su creador, es el primer diario argentino cien por ciento digital y, dentro de este estudio, el único que ofrece contenido totalmente gratuito, ya que Clarín y La Nación optaron por el modelo de muro poroso en abril y agosto de 2017, respectivamente. 
Esteban Zunino

Vivir con miedo: un estudio sobre las agendas digitales y las fuentes de información del delito, la violencia y el riesgo

\section{Fiabilidad}

Para establecer la fiabilidad de los datos, se recodificaron 150 piezas periodísticas (10\% de la muestra). El nivel de acuerdo medio se estimó mediante el coeficiente de correlación Rho de Spearman, ${ }^{3}$ el cual arrojó un resultado de $\rho=0,891$, aceptable para este tipo de trabajos.

\section{Resultados}

En primer lugar, se pretende determinar qué porción del hueco informativo (Budd, 1964) de las portadas de los medios digitales es ocupado por noticias que involucran algún tipo de delito, violencia o situaciones que ocasionen riesgos potenciales para la salud o la vida de las personas.

Los resultados, tomados de manera agregada, arrojan que el 23,5\% de las piezas informativas aluden de modo directo a este tipo de asuntos, lo que sustenta la primera hipótesis del estudio. Si se tienen en cuenta los datos de la Figura 1, es posible advertir que los tópicos asociados al delito, a la violencia y al riesgo ocupan diferentes posiciones en la agenda. Entre ellos se destacan los policiales (10,3\%) y los casos de corrupción (6,5\%). Entre los temas con visibilidad media se ubican la violencia de género $(1,8 \%)$ y la protesta social, habitualmente criminalizada y presentada en términos de perjuicios para el resto de la ciudadanía $(1,4 \%)$. Con menor visibilidad otra serie de acontecimientos se presentan como riesgos: amenazas ligadas a la salud $(0,8 \%)$, accidentes viales $(0,7 \%)$, siniestros urbanos o suburbanos no viales $(0,6 \%)$, incidentes medioambientales como incendios, inundaciones u otro tipo de catástrofes; asuntos vinculados con violaciones de derechos humanos $(0,4 \%)$, como casos de violencia institucional; y otras noticias vinculadas con el funcionamiento del Poder Judicial $(0,2 \%)$.

Una vez descripta la fisonomía general de las agendas mediáticas digitales y la incidencia en ellas de las noticias sobre delitos, violencia y riesgo, resulta interesante centrarse directamente en lo que podríamos denominar la "agenda del temor".

En términos generales, la mayoría de las noticias analizadas tienen un rasgo en común: una construcción discursiva dicotómica que diferencia a un protagonista de un antagonista: el victimario y la víctima. Sin embargo, su construcción discursiva depende del tema del que se trate. Los victimarios de los delitos asociados a lo que los mismos medios definen como "inseguridad" se construyen repitiendo el estereotipo del delincuente varón, joven y pobre que arremete intempestivamente y por razones individuales contra las clases medias urbanas, representación que reproduce la selectividad del sistema penal (Baratta, 2004). En cambio, los delitos de cuello blanco involu-

El Coeficiente de Spearman es una medida de correlación entre dos variables aleatorias continuas. El indicador oscila entre los valores de -1 y 1 . Un nivel de acuerdo aceptable es cuando el resultado es mayor a Rho de Spearman= 0,7 . 
Austral Comunicación

Volumen 9, número 2 (Diciembre de 2020): 553-582. ISSN 2313-9129

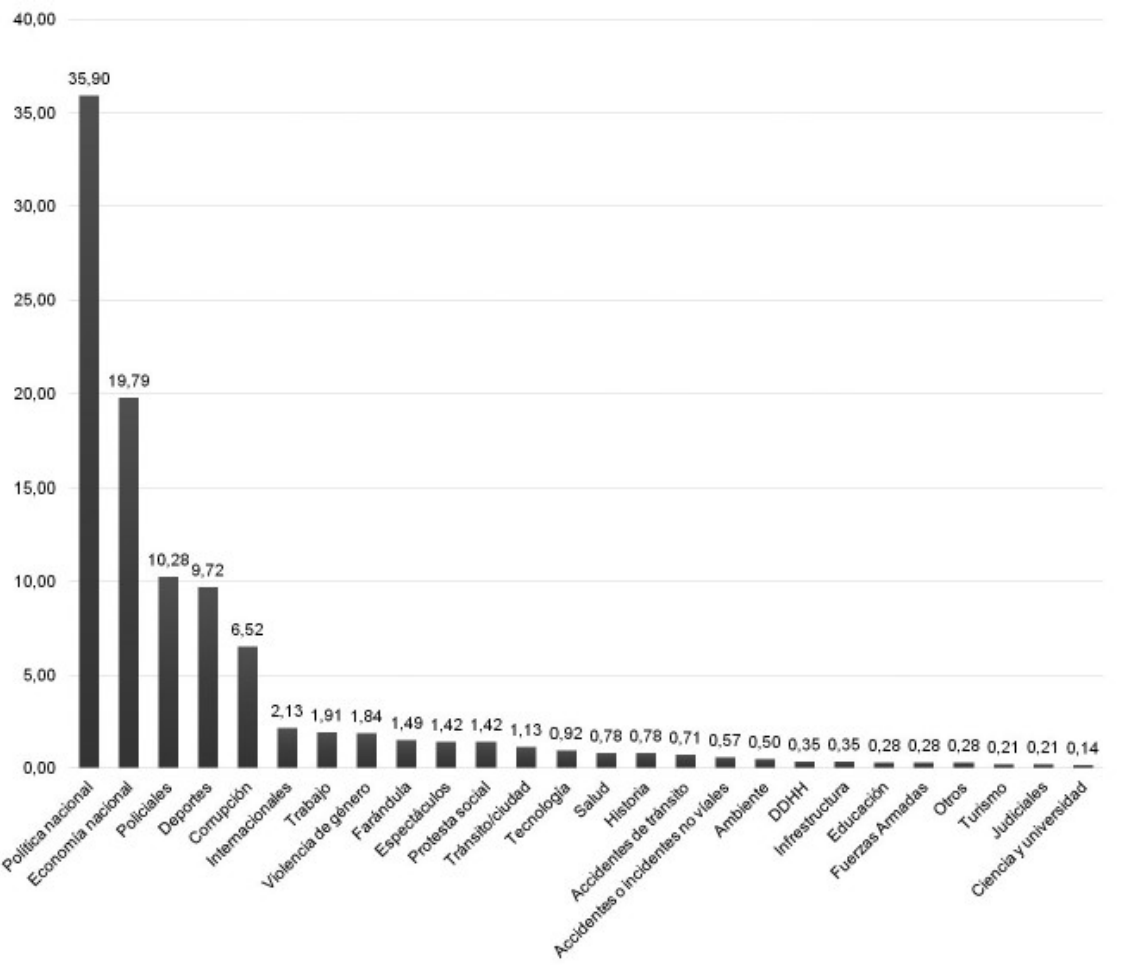

Figura 1. Frecuencia de tópicos de la agenda mediática. Clarín, La Nación e Infobae, abril-octubre de 2019. (Fuente: elaboración propia).

cran, por lo general, a funcionarios públicos que cometen ilícitos con plena conciencia y, amparados por el poder político, afectan a una víctima genérica o sujeto colectivo.

Sin embargo, una característica común en la construcción de ambos textos noticiosos es la condena mediática anticipada, es decir, la imputación de responsabilidades en casos sobre los que, al momento de la noticia, no suele haber condena judicial, lo que provoca una colisión entre el debate público y el principio de inocencia consagrado constitucionalmente.

Si se analizan únicamente las noticias ligadas al delito, a la violencia y al riesgo, los resultados totales de la frecuencia de cada uno de los tópicos en cada medio online analizado expresan cierta homogeneidad temática de las agendas informativas de los tres (Tabla 1). En términos agregados, los policiales clásicos son los tipos de noticias más frecuentes (40,5\%), a partir de las cuales suele construirse la noción de "inseguridad". Es decir, ilícitos contra la propiedad o las personas que tienen por caracetrística central su aleatoriedad (Kessler, 2009). 
Esteban Zunino

Vivir con miedo: un estudio sobre las agendas digitales y las fuentes de información del delito, la violencia y el riesgo

Tabla 1. Frecuencia de tópicos ligados al delito, a la violencia y al riesgo según cada medio. Clarín, La Nación e Infobae, abril-octubre de 2019. Fuente: elaboración propia

\begin{tabular}{lllll}
\hline & La Nación & Clarín & Infobae & Total \\
\hline Policiales & $38,10 \%$ & $35,90 \%$ & $45,80 \%$ & $40,50 \%$ \\
\hline Corrupción & $25,80 \%$ & $31,60 \%$ & $20,80 \%$ & $25,70 \%$ \\
\hline Trabajo & $17,50 \%$ & $17,10 \%$ & $7, \%$ & $13, \%$ \\
\hline Género & $4,10 \%$ & $3, \%$ & $12,50 \%$ & $7,30 \%$ \\
\hline Salud & $1 \%$ & $2,60 \%$ & $5, \%$ & $3, \%$ \\
\hline Accidentes de tránsito & $4,10 \%$ & $0,90 \%$ & $3,50 \%$ & $3, \%$ \\
\hline Accidentes o incidentes no viales & $3,10 \%$ & $3,40 \%$ & $0,70 \%$ & $2, \%$ \\
\hline Ambiente & $2,10 \%$ & $2,60 \%$ & $1,40 \%$ & $2 \%$ \\
\hline DDHH & $2,10 \%$ & & $2, \%$ & $1,40 \%$ \\
\hline Fuerzas Armadas & $2,10 \%$ & $0,90 \%$ & $0,70 \%$ & $1,10 \%$ \\
\hline Judiciales & & $1,70 \%$ & $0,70 \%$ & $0,80 \%$ \\
\hline
\end{tabular}

Algunos ejemplos de titulares de este tipo de noticias son: "Investigan la huella de Los Cuinis, los narcos mexicanos que se instalaron en Argentina" (Messi, 2019), "Balearon al diputado nacional Héctor Olivares en la esquina del Congreso y mataron a un hombre que lo acompañaba" (Balearon al diputado nacional..., 2019) y "El Gobierno reglamentó el uso de las armas Taser para las fuerzas de seguridad" (El Gobierno reglamentó..., 2019).

Los tres titulares seleccionados dan cuenta de algunas de las formas arquetípicas de las noticias policiales. El primero de ellos grafica el ingreso en agenda de uno de los tópicos de mayor relevancia y actualidad en América Latina, que comienza a cobrar un lugar de importancia en la Argentina: el narcotráfico (Rodríguez Alzueta, 2014; Sozzo, 2016). Este tema tiene la característica de haberse constituido en los últimos años en el principal promotor de un enemigo común en América Latina que suele legitimar estados de excepción que justifican el accionar represivo del Estado (Boiteux, 2017; Sánchez Sandoval, 2013).

El segundo titular está asociado con el homicidio del diputado nacional de la Provincia de La Rioja, Héctor Olivares, mientras caminaba frente al Congreso de la Nación. Dadas las características del caso -dramático, inesperado y que involucró a una persona importante del sistema político nacional-, su nivel de relevancia fue alto y fue seguido por los medios de comunicación por un lapso importante de tiempo, permaneciendo en lugares centrales de las agendas informativas durante todo el mes de mayo de 2019. Si bien al comienzo los medios promovieron la hipótesis de un asesinato motivado por razones políticas, esta fue desechada días después cuando se descubrió 
que el móvil estaba relacionado con asuntos ligados a la vida privada del legislador. Sin embargo, el homicidio contaba con todos los requisitos inherentes a la noticiabilidad: involucraba a una persona pública importante, fue inesperado, dramático y se dieron a conocer datos nuevos casi hora a hora con el transcurrir de la causa judicial, los cuales fueron cubiertos por los medios digitales de manera espectacularizada y en tiempo real.

Finalmente, el tercero de los titulares da cuenta de algo que fue habitual en la gestión gubernamental de Mauricio Macri (2015-2019): que el propio Gobierno genere discusiones sobre políticas públicas, punitivistas, que se conviertan luego en asuntos de agenda mediática (Galar y Focás, 2019). En este caso, la reglamentación de pistolas eléctricas para la contención de delitos urbanos.

Ahora bien, los casos de corrupción acapararon el 25,7\% de la agenda delictual. Sin embargo, estos fueron construídos discursivamente de manera diferente. El término más asociado a la palabra corrupción fue "kirchnerismo" -o simplemente "K"-, sustantivo que cumple la función de adjetivación de los casos más resonantes, al punto de que ambos términos se erigen como un par indisociable. Este tipo de construcción de equivalencias de un tema con un atributo se muestra potente en términos de transferencia entre medios y audiencias, ya que estas funcionan como una totalidad en la comprensión y recordación de los públicos en el largo plazo (McCombs y Valenzuela, 2014).

Partícipes necesarios, los empresarios vinculados a los principales procesos judiciales en curso no fueron habitualmente sindicados como victimarios, excepto aquellos ligados al kirchnerismo, denominados "empresarios K". Sobre ellos, los medios se mostraron más propensos a emitir sentencias que sobre otros muy importantes que, sin ser del riñón del pasado Gobierno, han admitido en sede judicial ser partícipes de sobornos entre 2003 y 2015.

Si bien estos casos fueron impulsados principalmente por importantes funcionarios del Poder Judicial, diferentes miembros del Poder Ejecutivo se han expresado ampliamente al respecto, incrementando la repercusión de la corrupción en los medios. "Cómo funcionaba el sistema de 'vuelos canillita' para que los Kirchner recibieran los diarios" (Cabot, 2019), "El patrimonio de Alberto Fernández, bajo la lupa: vive en un departamento de Pepe Albistur" (Ruiz, 2019) y "Las 19 propiedades del secretario de Néstor Kirchner que serán rematadas de forma 'inmediata"' (Las 19 propiedades..., 2019) son algunos de los titulares sobre el tema que vinculan casos de corrupción a exfuncionarios públicos kirchneristas.

La novedad sobre el tratamiento informativo de la corrupción fue que luego del 11 de agosto de 2019, fecha en la que se llevaron a cabo las elecciones Primarias Abiertas Simultáneas y Obligatorias que le dieron la victoria al candidato del Frente de Todos, 
Esteban Zunino

Vivir con miedo: un estudio sobre las agendas digitales y las fuentes de información del delito, la violencia y el riesgo

Alberto Fernández, las causas impulsadas por la justicia comenzaron a renovarse y, con ello, los posicionamientos mediáticos.

Así, aparecieron en agenda casos de supuesta corrupción cercanos al Gobierno de Macri que, aunque con menos visibilidad y calificaciones explícitas, también se incluyeron en las agendas informativas. "Anulan un fallo por el soterramiento del tren Sarmiento y favorecen a exfuncionarios K” (Salinas, 2019), “Correo Argentino: ordenan la intervención y envían el concurso a la Corte Suprema” (Alconada Mon, 2019) y "El empresario Daniel Vila denunció presiones del presidente Mauricio Macri" (El Empresario..., 2019) fueron algunos de los titulares que dan cuenta del "cambio de época" en el Poder Judicial y en los medios de comunicación en el tratamiento informativo de la corrupción. Cambio que se caracteriza por la aparición en agenda de temas ligados a la familia Macri, que adeuda el pago del canon por la gestión del Correo Argentino entre 1997 y 2003, y por la aparición de noticias sobre sobreseimientos y liberaciones de exfuncionarios kirchneristas y empresarios cercanos.

Otro de los asuntos centrales en las agendas informativas, con picos que se explican por la ocurrencia de eventos críticos (Pride, 1995), es la violencia de género. Si bien este tipo de casos en la actualidad son tipificados como tales, es decir, ya no se los suele titular como "crímenes pasionales" o simplemente "policiales", reciben un tratamiento también personalizado, descontextualizado y basado en el prototípico discurso del policial clásico.

Esto se vislumbra muy fuertemente en relación al tema de la violencia contra las mujeres y los femicidios. En ese orden, se extiende el hábito de la producción discursiva mediática basada en el consumo de morbo y narrativas sádicas que contribuyen a la construcción de imaginarios del terror, del pánico, del miedo (Gil et al., 2019, p. 48).

Algunos ejemplos de este tipo de titulares evidencian que los principales asuntos en agenda están relacionados con femicidios, violaciones y políticas públicas de corte punitivista. “'Te voy a partir el alma si no retirás la denuncia’: los audios que recibió la menor violada por una manada en Florencio Varela" (Fahsbender, 2019), "Femicidio en Misiones: hallaron degollada a una joven y vecinos filmaron a un hombre cuando salía del lugar del crimen" (Femicidio en..., 2019), “Implementarán medidas policiales inmediatas para frenar la violencia de género” (Gallo, 2019) son algunos de los titulares vinculados con este tópico.

Ahora bien, ¿por qué se incluyeron piezas relativas a la "protesta social" dentro de esta investigación? Se lo hizo porque la mayoría de las veces estas son presentadas por los medios como una amenaza contra los derechos y la seguridad del resto de las personas que no forman parte del colectivo que se moviliza. De este modo, se tiende a construir a un otro peligroso que es confrontado con un nosotros definido en términos de "gente", "vecino" o "ciudadano" merecedor de seguridad y resguardo de sus libertades 
individuales, entre ellas, el derecho al libre tránsito (Calzado, 2015). “Tras los disturbios, los piqueteros amenazan con acampar en Plaza de Mayo" (Tras los disturbios..., 2019), "El Gobierno volvió a calificar de 'extorsivos' los cortes de calle de las organizaciones sociales" (El gobierno volvió..., 2019), "Patricia Bullrich y el paro: 'Generan caos, miedo y riesgo país, con episodios cuasi terroristas”" (Patricia Bullrich..., 2019) son algunos ejemplos de los procedimientos mediáticos por los que suele criminalizarse la protesta social (Rodríguez Alzueta, 2014).

Finalmente, otro tipo de asuntos resultan menos relevantes en las agendas, pero también contribuyen con la construcción de un medio ambiente de temor y riesgos. Ejemplos de ello son la salud, los accidentes -sobre todo de tránsito- y las nuevas amenazas producto de catástrofes ambientales. "Alertan sobre los riesgos del uso de ibuprofeno: amplifica los efectos de bacterias como el estreptococo y agrava infecciones" (Alertan sobre..., 2019), "Otro trágico accidente en Carmen de Areco: ocho personas murieron tras un choque entre dos autos" (Otro trágico..., 2019), "Calentamiento global: parece que esta vez el lobo viene de verdad" (Vaca, 2019) son algunos ejemplos de los casos analizados, que, en conjunto con los asuntos más visibles, nos invitan cotidianamente a una agenda del riesgo y el temor.

En función de los objetivos planteados para este trabajo, resulta interesante analizar cuáles fueron los actores que lograron constituirse en legítimas fuentes de información de los tópicos más salientes, ya que estas ocupan un lugar privilegiado en la construcción de la noticia cuando se constituyen en portavoces eficaces que pueden promover no solo información a los medios, sino un punto de vista dominante sobre la situación mediatizada (Bennett, Lawrence y Livingston, 2007; Charron, 1995).

En primer lugar, la Tabla 2 permite constatar importantes niveles de correlación estadística entre las fuentes citadas por los tres medios analizados, lo que robustece la hipótesis anteriormente planteada de homogeneidad de las agendas. El dato constituye una primera señal de alarma en términos de calidad informativa, ya que la consonancia entre los portavoces de los diferentes medios confronta con el requisito de diversidad y pluralidad de voces (Pellegrini et al., 2011). Dado este hallazgo, el análisis de las fuentes de información se desarrollará de manera agregada con datos generales de los tres medios.

En términos generales, tal como lo expresa la Figura 2, las fuentes que dominaron la agenda mediática fueron el Poder Judicial (17,3\%), el Poder Ejecutivo (16\%), el entorno de las víctimas de delitos contra la propiedad y las personas $(14,9 \%)$ y las fuerzas de seguridad $(10,2 \%)$. Las empresas aparecen con un alto nivel de importancia como fuente $(6,8 \%)$, principalmente ligadas a casos de corrupción, al igual que dirigentes del kirchnerismo vinculados con esas mismas causas (2,9\%). La presencia de abogados en los medios es transversal a todos los tópicos $(4,8 \%)$. Sindicatos $(4,5 \%)$ y organizaciones 
Esteban Zunino

Vivir con miedo: un estudio sobre las agendas digitales y las fuentes de información del delito, la violencia y el riesgo

Tabla 2. Correlación de fuentes de información entre medios. Clarín, La Nación e Infobae, abril-octubre de 2019. Fuente: elaboración propia

\begin{tabular}{lllll}
\hline & & Fuentes La Nación & Fuentes Clarín & Fuentes Infobae \\
\hline \multirow{2}{*}{ Fuentes La Nación } & Correlación de Pearson & 1 & $0.953^{* *}$ & $0.953^{* *}$ \\
\cline { 2 - 5 } & Sig. (bilateral) & & 0 & 0 \\
\hline \multirow{2}{*}{ Fuentes Clarín } & Correlación de Pearson & $0.953^{* *}$ & 1 & $0.987^{* *}$ \\
\cline { 2 - 5 } & Sig. (bilateral) & 0 & $0.987^{* *}$ & 1 \\
\hline \multirow{2}{*}{ Fuentes Infobae } & Correlación de Pearson & $0.951^{* *}$ & 0 & 0 \\
\cline { 2 - 5 } & Sig. (bilateral) & 0 & & 0
\end{tabular}

** La correlación es significativa en el nivel 0,01 (bilateral).

sociales $(2,3 \%)$ se vinculan principalmente a delitos de obstrucción de calles en protestas. Finalmente, es marginal la presencia de científicos $(2,3 \%)$ y otros especialistas $(0,5 \%)$ en el cuerpo de las noticias, lo que contradice los requisitos básicos de calidad informativa (Pellegrini y Mujica, 2006). En tanto, en términos agregados, el 52,5\% de las fuentes fueron estatales, contra el $47,5 \%$ de las no estales, lo que corrobora la segunda hipótesis de este trabajo.

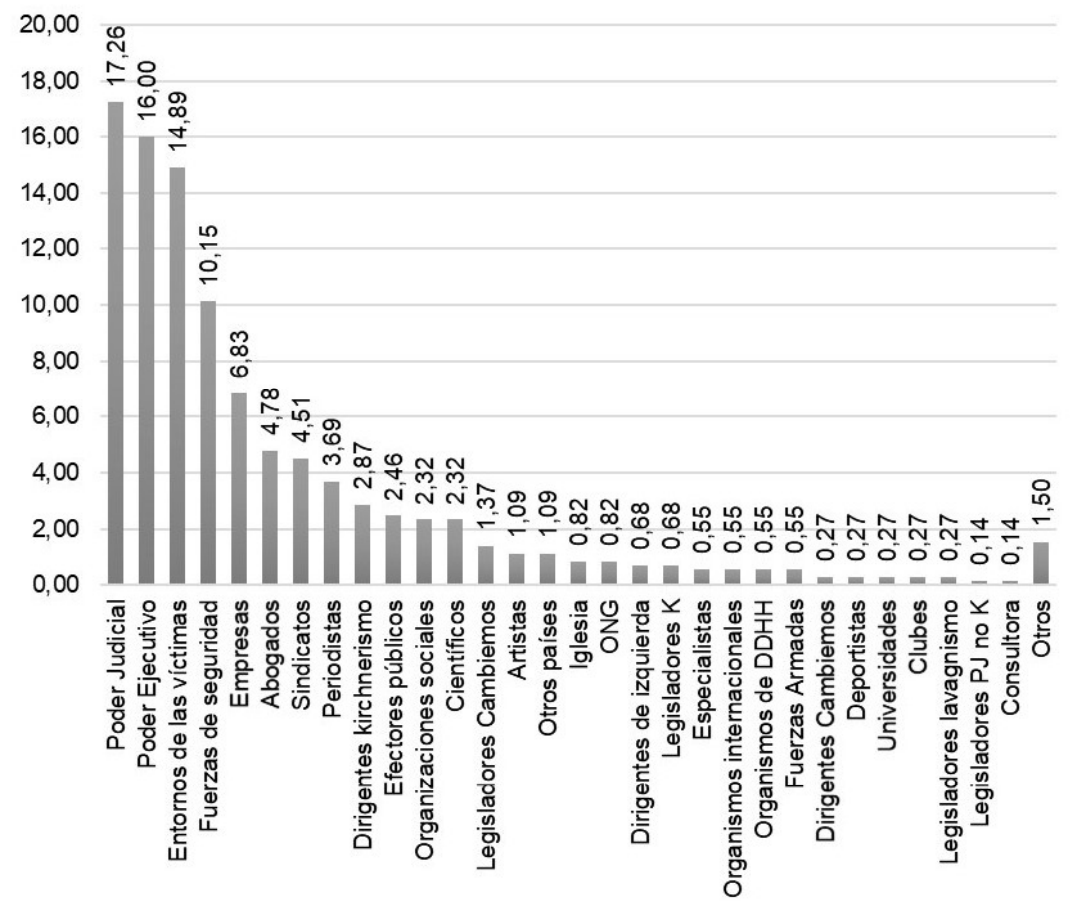

Figura 2. Frecuencia de fuentes en noticias sobre delito, violencia y riesgo. Clarín, La Nación e Infobae, abril-octubre 2019. (Fuente: elaboración propia). 
Austral Comunicación

Volumen 9, número 2 (Diciembre de 2020): 553-582. ISSN 2313-9129

Con el propósito de analizar la construcción de cada uno de los tópicos más relevantes de las agendas informativas, resulta oportuno identificar el despliegue de las fuentes de información y el crédito (Ferree et al., 2002) que estas obtienen en los cuatro tópicos principales, explicativos del $86,6 \%$ de las noticias sobre delitos, violencias y riesgos.

En el caso de los policiales clásicos, vinculados con delitos contra la propiedad o las personas, tienden a prevalecer las fuentes oficiales. Las fuerzas de seguridad, el Poder Judicial y el Poder Ejecutivo acaparan el 55,1\% de las menciones (Figura 3). Sin embargo, al tope del listado también aparecen los entornos de las víctimas, es decir, familiares, amigos, vecinos o testigos de los casos que se relatan son consultados asiduamente por los periodistas. Si bien estos actores suelen ser informantes clave a la hora de reconstruir los hechos, sus testimonios no se inscriben en una trama argumentativa que pretenda ir más allá del dato íntimo y escabroso.

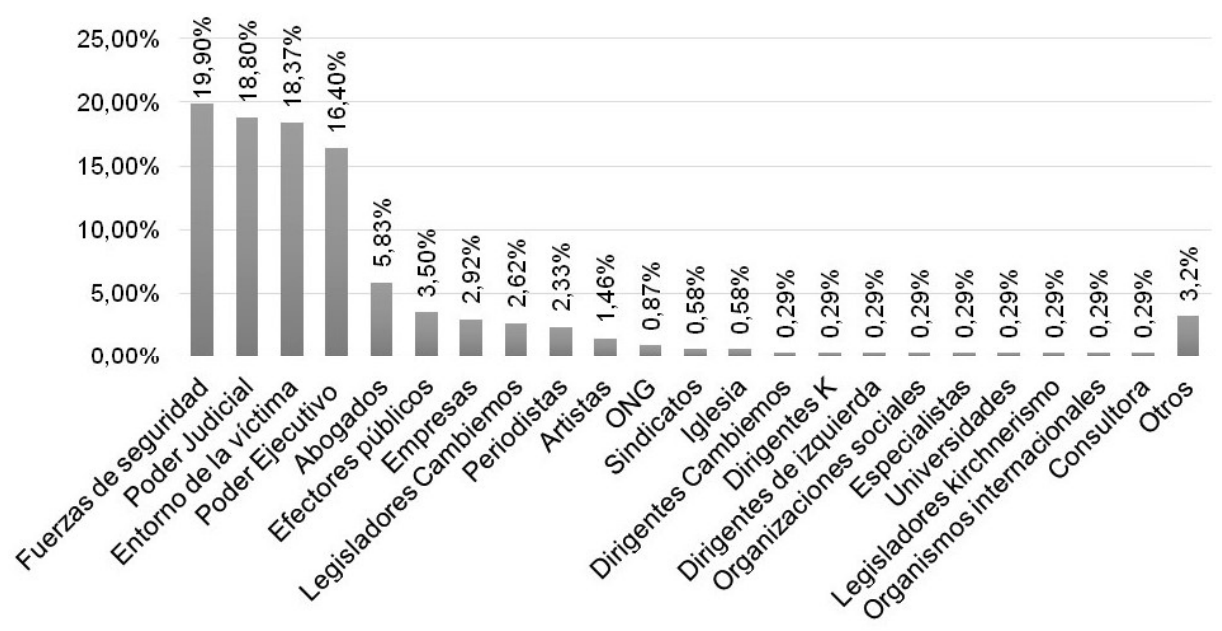

Figura 3. Frecuencia de fuentes en noticias sobre casos policiales. Clarín, La Nación elnfobae, abril-octubre de 2019. (Fuente: elaboración propia).

Tal como sostiene Vilker (2006), una de las características de la mediatización de la inseguridad es que desde la década del 90 los medios han puesto su mirada en la víctima. Esta situación permite problematizar algunas aristas. En primer lugar, las narraciones de los testimonios desgarradores de quienes sufrieron un ilícito violento contribuyen con la propagación de un estado de temor y victimización indirecta, ya que al tratarse de "gente común", refuerzan uno de los rasgos centrales de la construcción de la inseguridad: su aleatoriedad (Kessler, 2009). Por otro lado, tal como afirma Calzado (2015), una de las características posteriores a 2009 es una creciente "pérdida 
Esteban Zunino

Vivir con miedo: un estudio sobre las agendas digitales y las fuentes de información del delito, la violencia y el riesgo

de inocencia de la víctima" (p. 243), es decir, la puesta del foco del discurso mediático sobre causales asociadas a la víctima o su entorno que pudieran ser incluidas en el relato como factores explicativos de los hechos. Finalmente, los victimizados ocuparon un rol central en el discurso público securitario del Gobierno de Mauricio Macri. El enfoque del problema de seguridad desde el sufrimiento de quienes padecieron delitos fue propuesto por las principales autoridades estatales como un mecanismo legitimador de políticas punitivistas (Galar y Focás, 2019). En ese sentido, los altos niveles de frecuencia de las víctimas y/o sus entornos como fuentes, además de contribuir con la dramatización y espectacularización de los hechos, resultan coherentes con el discurso y encuadre propuesto por los funcionarios gubernamentales.

Un dato extra que aporta luz al análisis es el nivel de crédito que obtienen las principales voces citadas. Este se estipula a partir de una categorización construida para estimar, además de la presencia de la fuente en la nota, si el medio acredita o pone en duda el punto de vista del actor citado. De este modo, se estima, además de la frecuencia de cada fuente en las coberturas informativas, el nivel diferencial de crédito (standing) que estas obtienen.

La Figura 4 evidencia que las principales fuentes incluidas en las noticias policiales no solo obtienen altos niveles de visibilidad, sino que son prácticamente incuestiona-

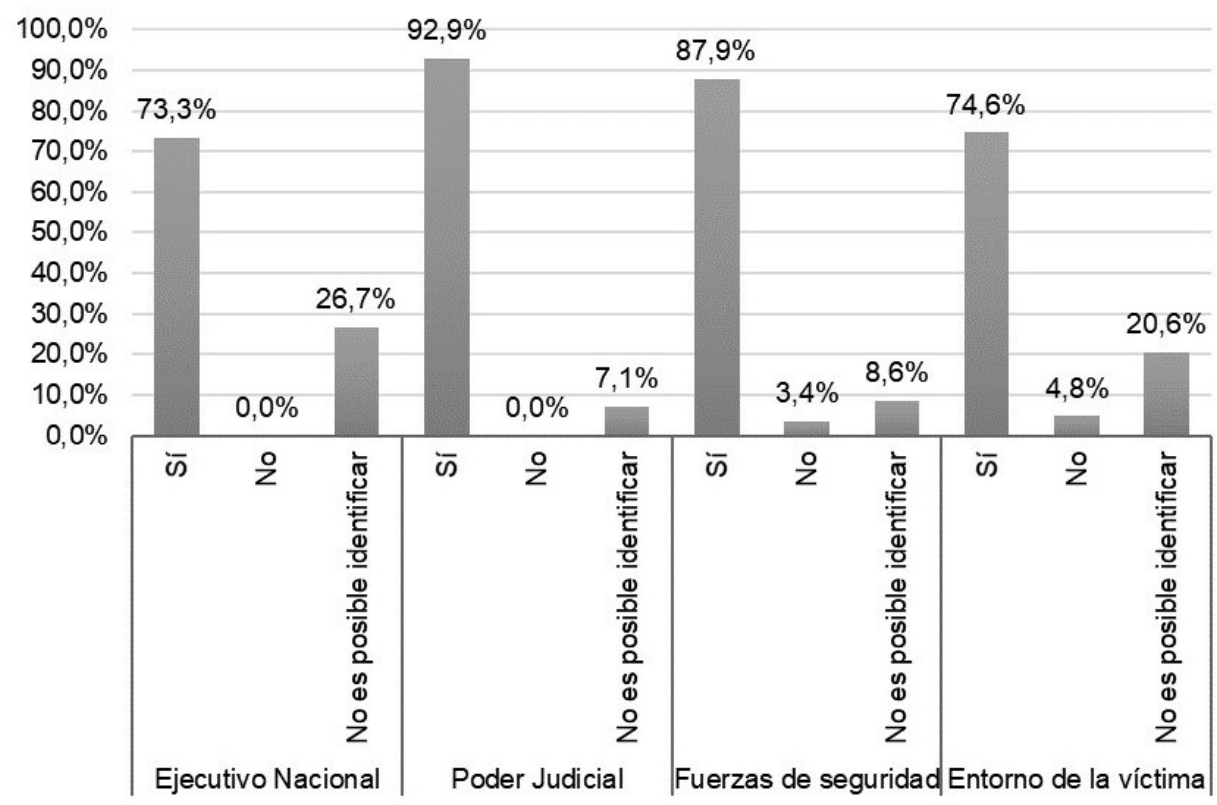

Figura 4. Crédito de las principales fuentes en noticias sobre casos policiales. Clarín, La Nación e Infobae, abril-octubre de 2019. (Fuente: elaboración propia). 
Austral Comunicación

Volumen 9, número 2 (Diciembre de 2020): 553-582. ISSN 2313-9129

bles para los medios, al menos en dos sentidos. En primer lugar, porque tal como se puede ver en las frecuencias, sobre todo las fuentes estatales monopolizan los puntos de vista presentes en los medios. En segundo lugar, porque en lo que a estas respecta, el nivel de crédito que obtienen sus versiones es muy alto ( $84,7 \%$ promedio). Finalmente, el standing obtenido por las versiones que deslizan los entornos de las víctimas es algo menor, pero también importante $(74,6 \%)$.

Lo anterior expresa que la noticia policial se basa generalmente en una serie de acontecimientos dramáticos que son fuertemente impulsados a través de los medios por las fuentes oficiales y que, en el mejor de los casos, son contrastados por el entorno de la víctima, recayendo la mayoría de las veces en una cobertura episódica (Iyengar, 1990), dramática (Martini, 2017) y descontextualizada de sus factores determinantes estructurales (Bennett, 1990). Prueba de ello es la presencia marginal de especialistas consultados por los medios de comunicación $(0,3 \%)$, quienes podrían aportar nuevas perspectivas al relato.

Si seguimos adelante con el estudio de los delitos más relevantes en las agendas investigadas, la corrupción ocupa un lugar central, aunque se construye a partir de lógicas diferentes. Y eso también se visibiliza al nivel de la presencia diferencial de las fuentes de información.

Tal como se desprende de la Figura 5, sobre este tópico también dominan las fuentes estatales, aunque se destaca fuertemente el Poder Judicial, encargado de tramitar las causas $(33,8 \%)$.

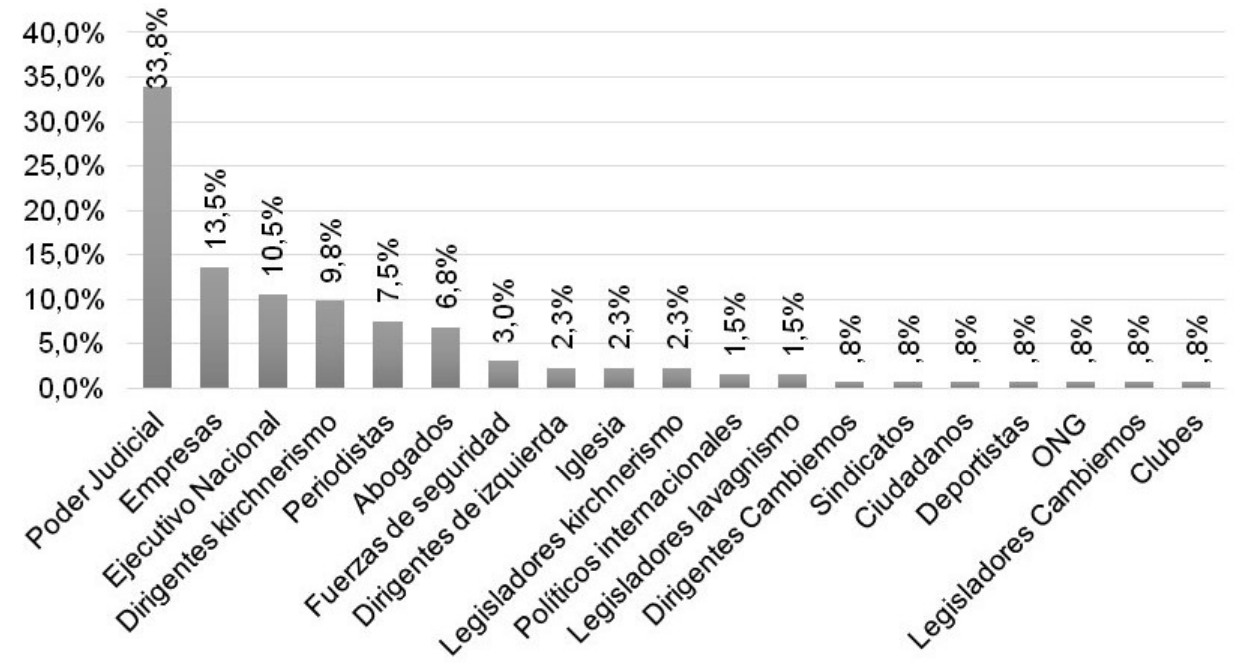

Figura 5. Frecuencia de fuentes en noticias sobre corrupción. Clarín, La Nación e Infobae, abril-octubre de 2019. (Fuente: elaboración propia). 
Esteban Zunino

Vivir con miedo: un estudio sobre las agendas digitales y las fuentes de información del delito, la violencia y el riesgo

Como se sostuvo previamente, las principales acusaciones presentes en este tipo de noticias remiten a un supuesto entramado de coimas y sobreprecios que los Gobiernos de Néstor Kirchner y de Cristina Fernández (2003-2015) habrían establecido con algunas empresas muy importantes del país y de la región. Por lo tanto, no llama la atención que en segundo lugar se ubiquen las empresas como portavoces del caso (13,5\%). En tercer lugar, es importante la presencia del Poder Ejecutivo como fuente (10,5\%), ya que, de signo opositor al kirchnerismo, fue el encargado de impulsar y sostener el tema en los medios de comunicación a partir, sobre todo, de declaraciones de funcionarios sobre las diferentes causas que involucraban a sus adversarios políticos. Igualmente, adquieren importante visibilidad como fuentes los propios dirigentes del kirchnerismo, acusados mediáticamente de ser responsables de actos de corrupción en el manejo del Estado (9,8\%). Por último, también es importante la aparición de periodistas como informantes centrales de las noticias (7,5\%). La explicación es que muchos de estos casos surgieron de investigaciones periodísticas o filtraciones de información de los poderes Ejecutivo y/o Judicial a ciertos medios de comunicación, los cuales luego fueron citados por otros medios en un claro ejemplo de intermedia agenda setting (Meraz, 2011).

Finalmente, un dato central de la estructura de este tipo de noticias es la construcción de una víctima ambigua, que si bien recae en la ciudadanía (las víctimas son "los ciudadanos"), no suele ser interpelada ni consultada en el tratamiento mediático, a diferencia del policial clásico. De allí su prácticamente nula aparición como fuente de información $(0,8 \%)$.

La Figura 6 pone en evidencia que sobre "corrupción" el crédito y descrédito de las fuentes está fuertemente polarizado. Mientras que la totalidad de las versiones del Poder Ejecutivo y siete de cada diez de las del Poder Judicial y de las de los periodistas son respaldadas por los medios, los puntos de vista promovidos por dirigentes del kirchnerismo son explícitamente rechazados en la mayoría de los casos, sin hallarse ningún apoyo mediático a sus dichos. En tanto, las apreciaciones expresadas por las empresas, contraparte necesaria de cualquier esquema de corrupción, son evaluadas de manera más ambigua por los medios, dependiendo en buena medida de qué empresarios se trate. Mientras que la mayoría de las versiones suscitadas por empresas cercanas a Cristina Fernández y/o a los exfuncionarios de su Gobierno son rechazadas, otras provenientes de empresarios cercanos a Mauricio Macri y/o a su entorno son acreditadas o no puestas en duda explícitamente.

Tal constatación permite ratificar la suposición de Charrón (1995) acerca de que la mera visibilidad de la fuente no es suficiente para inferir su influencia sobre el punto de vista propuesto por el medio. El análisis de las noticias sobre corrupción en los portales Clarín, La Nación e Infobae demuestra que algunos actores importantes del 
Austral Comunicación

Volumen 9, número 2 (Diciembre de 2020): 553-582. ISSN 2313-9129

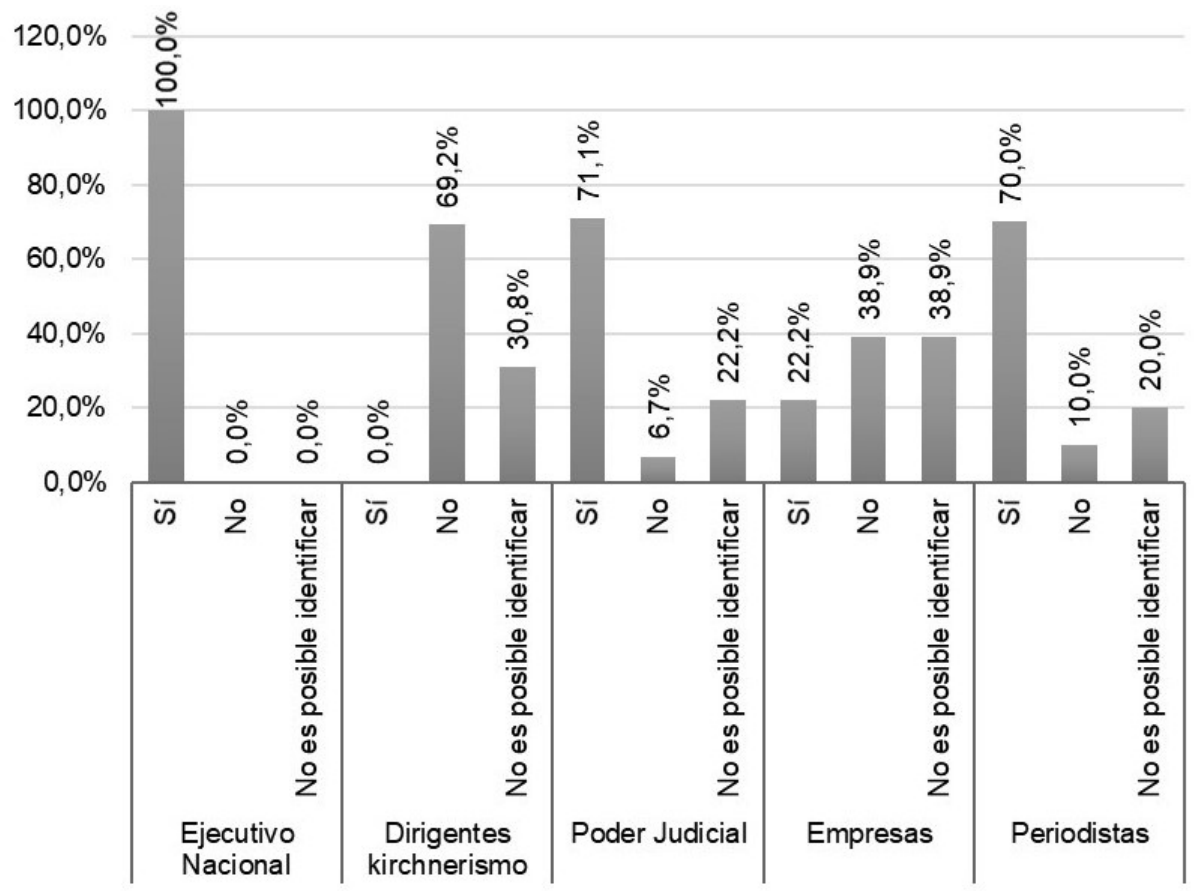

Figura 6. Crédito de las principales fuentes en noticias sobre corrupción. Clarín, La Nación e Infobae, abril-octubre 2019. (Fuente: elaboración propia).

kirchnerismo fueron asiduamente consultados por la prensa, lo que les otorgó visibilidad en las noticias, aunque no crédito.

Prosiguiendo en el camino de desandar la construcción mediática de la violencia, los riesgos y la inseguridad, es de destacar que la protesta social haya sido presentada por los medios mucho más en términos de perjuicios y riesgos para la ciudadanía que a partir de los motivos que la generan o de las soluciones que podrían desactivarla

Si bien ya se problematizaron sus representaciones, tendientes a la criminalización de quienes la promueven y de la construcción de las manifestaciones como amenazas para el bien común, el análisis de las fuentes echa luz y funciona como complemento para esa interpretación. La Figura 7 permite analizar que, a diferencia del resto de los ilícitos más recurrentes en las agendas, en la cobertura de la protesta social predominaron las fuentes no estatales. Entre ellas, las organizaciones sociales (40\%) y los sindicatos $(17,5 \%)$ fueron las más habituales. El Poder Ejecutivo, principal promotor de la perspectiva criminalizante, aparece también con un alto nivel de visibilidad (30\%). Le siguen las fuerzas de seguridad encargadas de los operativos disuasivos o abiertamente represivos (5\%). 
Esteban Zunino

Vivir con miedo: un estudio sobre las agendas digitales y las fuentes de información del delito, la violencia y el riesgo

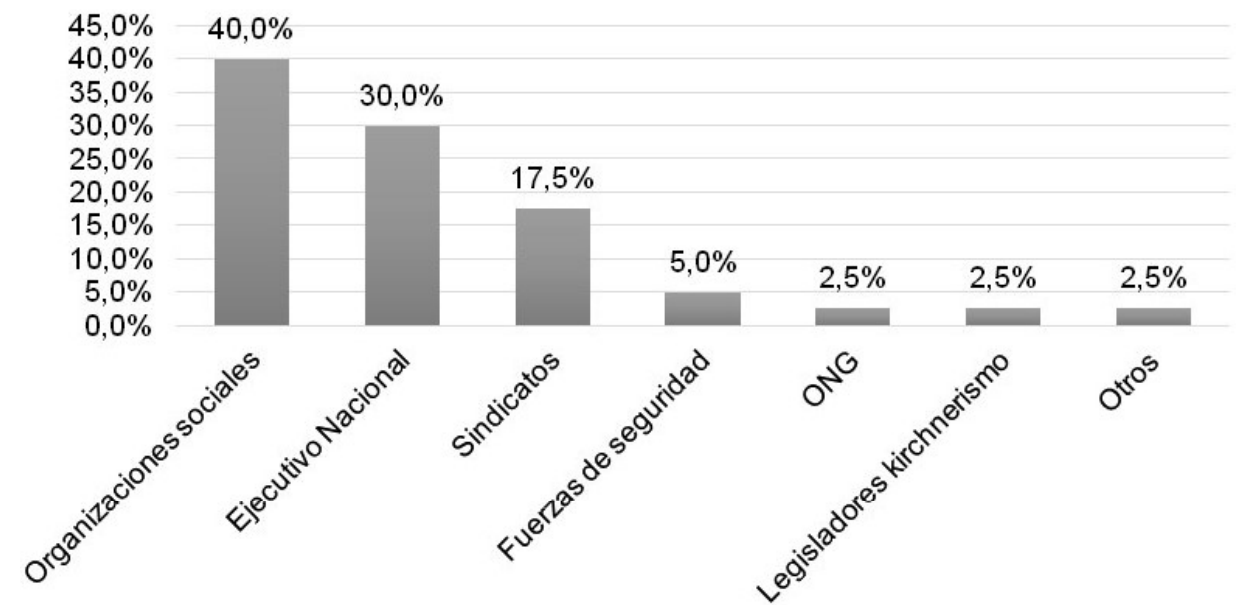

Figura 7. Frecuencia de fuentes en noticias sobre protesta social. Clarín, La Nación e Infobae, abril-octubre de 2019. (Fuente: elaboración propia).

Si se analiza el standing diferencial de cada una de ellas, se podría decir, con el apoyo de los datos de la Figura 8, que el alto nivel de crédito de las fuerzas de seguridad (100\%) y del Poder Ejecutivo (66,6\%) contrasta con un importante grado de descrédito de los portavoces de los movimientos sociales y sindicatos que impulsaron las medidas de lucha (71,4\% y $31,3 \%$, respectivamente), con casi nulos porcentajes de aceptación de sus puntos de vista y explicaciones. Ambos hallazgos ponen de manifiesto que aunque las fuentes no oficiales dominaron en términos de visibilidad, sus voces fueron permanentemente desacreditadas por los medios de comunicación. En cambio, con menor presencia mediática en relación con las primeras, los funcionarios del Poder Ejecutivo y de las fuerzas represivas lograron instalar sus argumentos criminalizantes como válidos en las coberturas.

Finalmente, se analizan las frecuencias de aparición de fuentes de los casos relacionados con violencia de género en los principales medios digitales del país.

Uno de los datos que este estudio permite vislumbrar es que los sucesos de violencia contra las mujeres y minorías de género y los femicidios suelen ser tipificados con las etiquetas que les corresponden, lo que los aleja de su inclusión periodística dentro del policial clásico. Sin embargo, más allá de su correcta identificación, la construcción de estas noticias no suele escapar a las formas clásicas de las secciones policiales, en las cuales muchas siguen siendo incluidas (Gil et al., 2019).

La Figura 9 permite percibir que entre las fuentes de estas piezas sobresalen los entornos de las víctimas $(44,6 \%)$. La indagación periodística al testigo directo o entorno cercano logra incorporar datos escabrosos a la información, lo que acarrea una 
Austral Comunicación

Volumen 9, número 2 (Diciembre de 2020): 553-582. ISSN 2313-9129

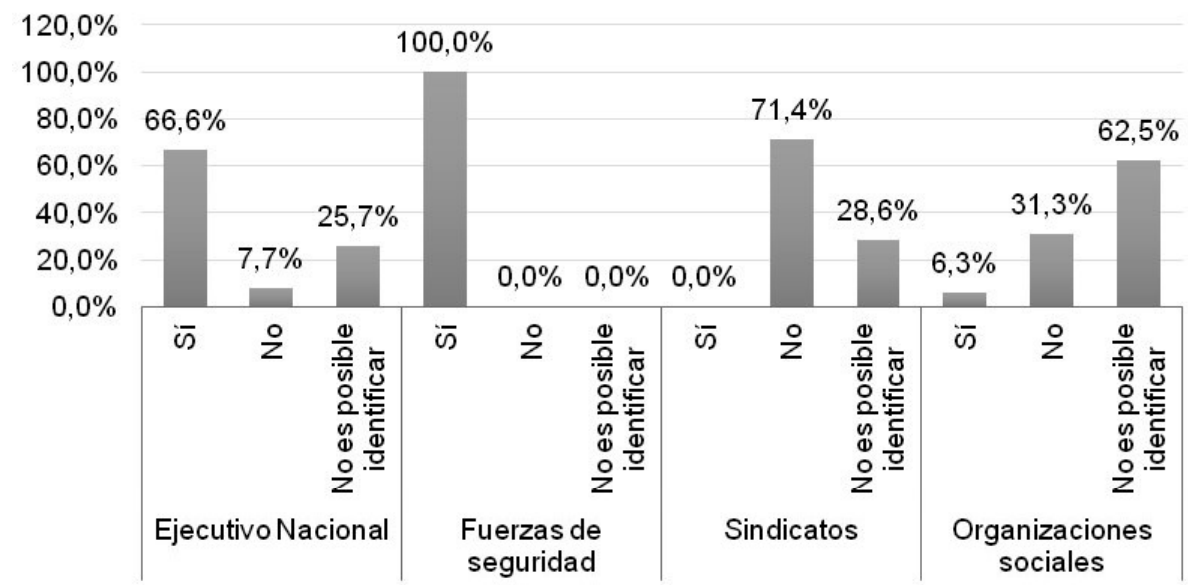

Figura 8. Crédito de las principales fuentes en noticias sobre protesta social. Clarín, La Nación e Infobae, abril-octubre de 2019. (Fuente: elaboración propia).

consecuencia aún mayor. A partir de este tratamiento mediático, los casos son inscriptos en el ámbito privado, desligando los sucesos de sus determinantes estructurales relacionados con la cultura heteropatriarcal, las diferencias sexo-genéricas y de clase y las violencias que de estos factores se desprenden. En consecuencia, el Estado aparece desresponsabilizado, ya que los sucesos quedan circunscriptos al ámbito doméstico. Tal es así que el Poder Judicial (14,3\%) y el Poder Ejecutivo (9,1\%) adquieren una visibilidad menor que en el resto de los delitos analizados.

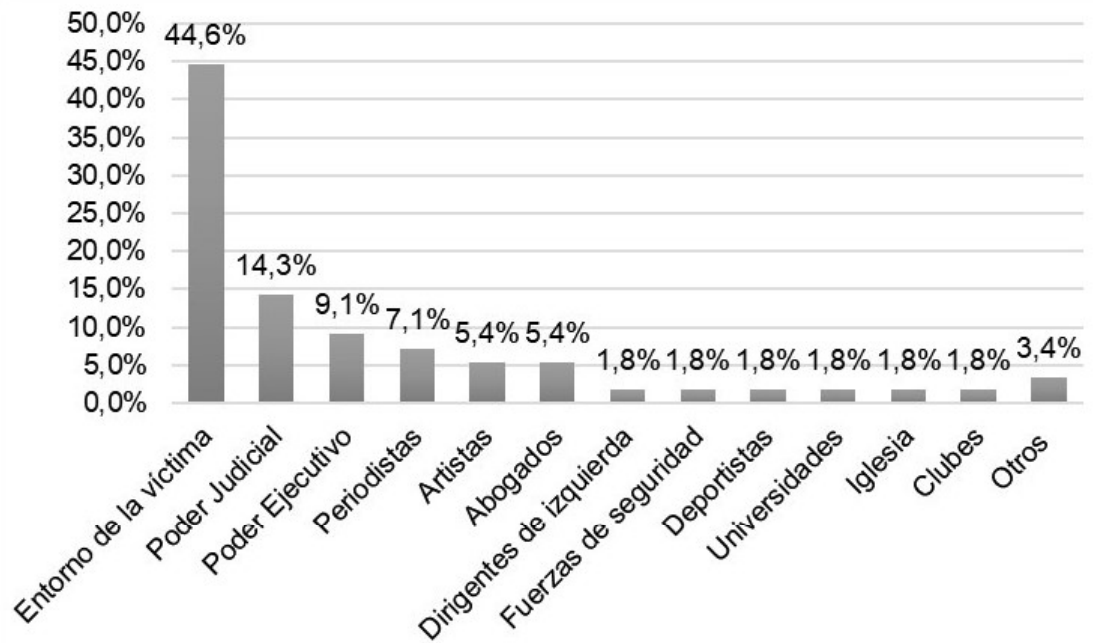

Figura 9. Frecuencia de fuentes en noticias sobre violencia de género. Clarín, La Nación e Infobae, abril-octubre de 2019. (Fuente: elaboración propia). 
Esteban Zunino

Vivir con miedo: un estudio sobre las agendas digitales y las fuentes de información del delito, la violencia y el riesgo

Sobre estos acontecimientos, es importante destacar la usual aparición de periodistas $(7,1 \%)$ y artistas $(5,4 \%)$ como fuentes. Se trata, sobre todo, de mujeres periodistas y artistas que han organizado colectivos que fueron muy importantes para la difusión de casos concretos y para el seguimiento de la problemática en los medios de comunicación. Finalmente, la mirada de expertos con perspectiva de género aparece absolutamente invisibilizada.

Para finalizar, resulta interesante advertir que sobre este tópico en particular la mayoría de las fuentes consultadas obtienen crédito en sus afirmaciones (ver Figura 10). Los casos que muestran mayor nivel de descrédito remiten a periodistas (25\%) y están ligados a declaraciones de trabajadores de prensa que fueron acusados directamente por ejercer violencia de género contra colegas. Finalmente, el pequeño nivel de descrédito a las versiones del entorno de las víctimas (4\%) remite a asuntos en los que ese mismo entorno estuvo sospechado de obstruir el desarrollo de las investigaciones por ser el victimario o estar relacionado con él.

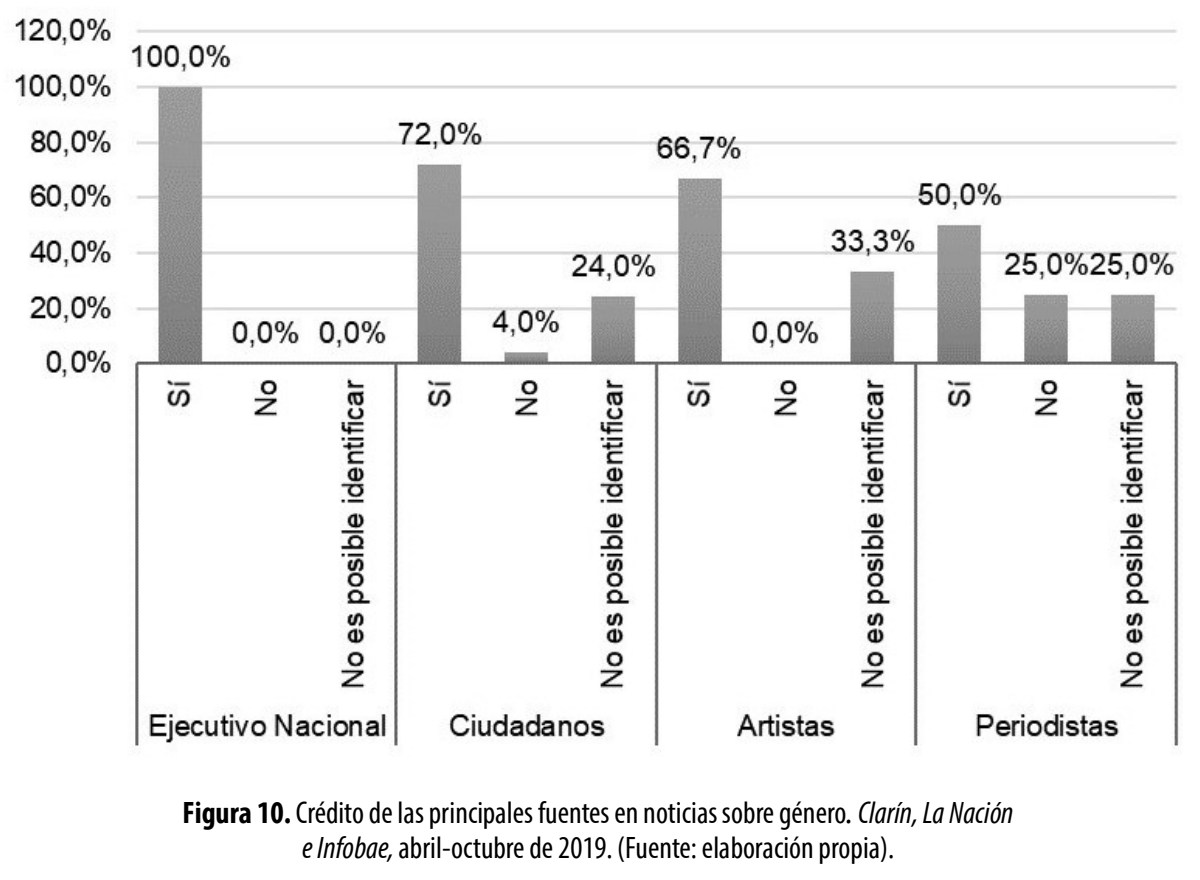

\section{Conclusiones}

Este trabajo analizó la composición y fisonomía de la agenda mediática de los principales portales online del país, poniendo especial atención en los casos que remiten a noticias sobre delitos, violencias y riesgos. 
Austral Comunicación

Volumen 9, número 2 (Diciembre de 2020): 553-582. ISSN 2313-9129

Una primera constatación al respecto es que un cuarto de las agendas digitales están compuestas por este tipo de informaciones, cifra que se eleva si se tuvieran en cuenta las piezas sobre los mismos tópicos que ocurren fuera del ámbito nacional, lo que refuerza la primera hipótesis de esta investigación. En ese sentido, resulta posible afirmar que, dado su dramatismo e imprevisibilidad, el delito y la violencia suelen ser muy visibles en la superficie mediática y despiertan, desde hace décadas, interés público (Bonilla y Rincón, 1998; Calzado, 2015; Dammert, 2010; Martini, 2007). En este estudio, los tópicos más salientes fueron los policiales, la corrupción, la protesta social criminalizada y los delitos de género.

En términos generales, todas las noticias analizadas mantienen la estructura de un protagonista y un antagonista que se construyen como víctima y victimario. $Y$ esa representación maniquea se traslada al uso de las fuentes. Así, queda en evidencia que tienden a prevalecer únicamente los puntos de vista de actores estatales -Poder Judicial, Poder Ejecutivo y fuerzas de seguridad- y del entorno de las víctimas. Estos últimos, consultados habitualmente en contextos de emoción violenta, suelen expresarse en concordancia con las visiones oficiales, sobre todo en lo que hace a las soluciones propuestas, casi siempre de corte punitivista.

Sin embargo, los diferentes tópicos promueven construcciones diversas de víctimas y victimarios. Mientras que en los policiales y en los delitos de género la responsabilización recae en la intención y acción de individuos aislados, desacoplando las problemáticas de sus factores explicativos estructutrales y contextuales (Martini, 2017), en las noticias sobre corrupción y criminalización de la protesta se construye un victimario colectivo y organizado, presentado en términos de asociación ilícita o banda que arremete contra bienes públicos y derechos constitucionales de una víctima también colectiva, pero abstracta, construída a partir de colectivos de identificación como la ciudadanía, los vecinos o simplemente la gente. En ambos casos, la ausencia de voces de especialistas o de actores con perspectivas diversas que puedan anclar los problemas en contextos explicativos más amplios da cuenta de una cobertura episódica, simplifificada y maniquea.

Siguiendo la segunda hipótesis de este trabajo, se corrobora una mayor presencia de fuentes oficales estatales (52,5\%), aunque se añaden algunos hallazgos dignos de ser revisados. Como se dijo, mientras que el Poder Judicial, las fuerzas de seguridad y el Poder Ejecutivo dominan los asuntos policíacos, es notorio cómo en estos y en los delitos de género cobra cada vez mayor importancia la voz del entorno de la víctima. Su presencia fomenta la presentación de las historias privadas que rodean a los casos y aportan altas dosis de condimentos sensacionalistas.

En cambio, en los delitos de corrupción y en la criminalización de la protesta social la situación es diferente. En las primeras dominan las voces de los actores estatales, 
Esteban Zunino

Vivir con miedo: un estudio sobre las agendas digitales y las fuentes de información del delito, la violencia y el riesgo

con una aparición importante de la palabra de los supuestos victimarios, aunque siempre desacreditada. En las segundas, las organizaciones sociales y los sindicatos son los más citados, aunque son presentados como victimarios de delitos contra el patrimonio público y el derecho al tránsito, por lo cual, sus visiones no logran pasar el filtro del crédito mediático. Finalmente, ambos tipos de noticias tienen un rasgo común: la construcción de un victimario colectivo y organizado que atenta contra una víctima también colectiva y abstracta. En el primer caso se trata de los sindicatos y las organizaciones sociales contra "la gente"; en el segundo, de los políticos en contra de los intereses del mismo colectivo de identificación. Tal construcción, carente de matices y problematización, redunda en un profundo descrédito de la actividad política en general, lo que contribuye con una creciente espiral de cinismo (Aruguete, 2013; Cappella y Jamieson, 1996).

El estudio aquí desarrollado se presenta como una primera aproximación general a la construcción de las noticias sobre delito, violencia y riesgo en los medios digitales más leídos de la Argentina. Su principal potencia es haber ido más allá de la indagación sobre la "inseguridad" -ampliamente investigada- que suele involucrar solo a una porción de los delitos y violencias que se incluyen cotidianamente en las agendas informativas. Como se observó, la comparación entre la construcción de los diferentes tópicos de la agenda del temor invita a complejizar el análisis sobre cómo se construyen desde los medios aquellas situaciones que nos ponen en riesgo. Tal como se constató, si bien prevalece una estructura dicotómica y maniquea en la cimentación de las noticias, basada en víctimas y vitimarios presentados en términos de buenos y malos, la representación de cada uno de ellos es diferente según el caso, como lo son los actores involucrados y los promotores de visiones, explicaciones y soluciones. Queda el desafío de indagar con mayor profundidad sobre las particularidades de cada uno de los tópicos, incorporando nuevas dimensiones teóricas y metodológicas para la construcción de los datos y su interpretación.

\section{Referencias}

Albornoz, L. (2006). Periodismo digital. Los grandes diarios en la red ( $1^{\mathrm{a}}$ ed.). Buenos Aires: La Crujía.

Alconada Mon, H. (11 de septiembre de 2019). Correo Argentino: ordenan la intervención y envían el concurso a la Corte Suprema. La Nación. Obtenido de https://www.lanacion.com.ar/politica/ correo-argentino-ordenan-intervencion-envian-concurso-corte-nid2286791.

Alertan sobre los riesgos del uso de ibuprofeno: amplifica los efectos de bacterias como el estreptococo y agrava infecciones. (20 de abril de 2019). Infobae. Obtenido de 
Austral Comunicación

Volumen 9, número 2 (Diciembre de 2020): 553-582. ISSN 2313-9129

https://www.infobae.com/america/ciencia-america/2019/04/20/francia-lanzauna-seria-advertencia-sobre-el-uso-del-ibuprofeno-puede-agravar-anginasvaricela-y-otras-infecciones/.

Anitua, G. (2010). Historias de los pensamientos criminológicos ( $1^{\mathrm{a}}$ ed.). Buenos Aires: Didot.

Argentina: Infobae reafirma su liderazgo entre los medios digitales. (2 de mayo de 2019). Prensario Internacional. Obtenido de https://prensario.tv/novedades/3137argentina-infobae-reafirma-su-liderazgo-entre-los-medios-digitales.

Aruguete, N. (2013). La narración del espectáculo político: pensar la relación entre sistema de medios y poder político. Austral Comunicación, 2(1), 205-216. Obtenido de https://doi.org/10.26422/aucom.2013.0202.aru.

Ávila, K. (2016). Homicidios en Venezuela: principales debates y aproximaciones explicativas. Misión Jurídica, 12, 117-140.

Bagdikian, B. H. (1985). The U. S. Media Supermarket or Assembly Line? Journal of Communication, 3(35), 97-109. Obtenido de https://doi.org/https://doi. org/10.1111/j.1460-2466.1985.tb02451.x.

Balearon al diputado nacional Héctor Olivares en la esquina del Congreso y mataron a un hombre que lo acompañaba. (9 de mayo de 2019). Infobae. Obtenido de https://www.infobae.com/sociedad/policiales/2019/05/09/balearon-al-diputadonacional-hector-olivares-en-la-esquina-del-congreso-y-mataron-a-un-hombreque-lo-acompanaba/.

Baratta, A. (2004). Criminología y sistema penal.Compilación in memoriam ( $1^{a}$ ed.). Buenos Aires: Euros Editores.

Becerra, M. (2015). De la concentración a la convergencia. Políticas de medios en Argentina y América Latina ( $1^{\mathrm{a}}$ ed.). Buenos Aires: Paidós.

Bennett, W. L. (1990). Toward a Theory of Press-State Relations in the United States. Journal of Communication, 40(2), 103-127. Obtenido de https://doi. org/10.1111/j.1460-2466.1990.tb02265.x.

Bennett, W. L. (1991). News: The Politics of Illusion, Ninth Edition (1 ${ }^{\mathrm{a}}$ ed.). Nueva York: Longman.

Bennett, W. L., Lawrence, R. G. y Livingston, S. (2007). When the Press Fails. Political Power and The News Media from Iraq to Katrina ( $1^{\text {a }}$ ed.). Chicago/Londres: The University of Chicago Press.

Boiteux, L. (2017). Brasil: las cárceles de la droga y de la miseria. Nueva Sociedad, 268(marzo-abril), 14-22.

Bonilla, J. y Rincón, O. (1998). Violencia en la pantalla: televisión, jóvenes y violencia en Colombia. Diálogos de la Comunicación, 53, 37-50. Obtenido de https://doi. org/10.1017/CBO9781107415324.004. 
Esteban Zunino

Vivir con miedo: un estudio sobre las agendas digitales y las fuentes de información del delito, la violencia y el riesgo

Brosius, H. B. y Eps, P. (1995). Prototyping through Key Events: News Selection in the Case of Violence Against Aliens and Asylum Seekers in Germany. European Journal of Communication, 10(3), 391-412. Obtenido de https://doi.org/10.1177/0 267323195010003005.

Budd, R. W. (1964). U.S. News in the Press Down Under. The Public Opinion Quarterly, 28(1), 39-56.

Cabot, D. (14 de abril de 2019). Cómo funcionaba el sistema de "vuelos canillita" para que los Kirchner recibieran los diarios. La Nación. Obtenido de https://www. lanacion.com.ar/politica/como-funcionaba-el-sistema-de-vuelos-canillita-paraque-los-kirchner-recibieran-los-diarios-nid2238132.

Calzado, M. (2012). Ciudad segura. Vecindad, víctimas y gubernamentalidad. Notas sobre la campaña electoral del PRO en la Ciudad de Buenos Aires (2011). VII Jornadas de Sociología de la Universidad Nacional de La Plata "Argentina en el escenario latinoamericano actual: debates desde las ciencias sociales". La Plata, Argentina, 5 al 7 de diciembre de 2012.

Calzado, M. (2015). Inseguros. El rol de los medios y la respuesta política frente a la violencia de Blumberg a hoy ( $1^{\mathrm{a}}$ ed.). Buenos Aires: Aguilar.

Cappella, J. N. y Jamieson, K. H. (1996). News Frames, Political Cynicism, and Media Cynicism. Annals AAPSS, 546, 71-84.

Charron, J. (1995). Los medios y las fuentes. Los límites del modelo de agenda setting. En Gilles Gauthier, M. J. (ed.). Comunicación y Política (1 ${ }^{\text {a }}$ ed., pp. 72-93). Barcelon: Gedisa.

Dammert, L. (2010). Crimen e inseguridad. Politicas, temas y problemas en las américas ( $1^{a}$ ed.). Santiago de Chile: Catalonia/Flacso.

Entel, A. (2007). La ciudad y los miedos. La pasión restauradora ( $1^{\mathrm{a}} \mathrm{ed}$.). Buenos Aires: La Crujía.

El empresario Daniel Vila denunció presiones del presidente Mauricio Macri. (19 de septiembre de 2019). Infobae. Obtenido de https://www.infobae.com/ politica/2019/09/19/el-empresario-daniel-vila-denuncio-presiones-delpresidente-mauricio-macri/.

El Gobierno reglamentó el uso de las armas Taser para las fuerzas de seguridad. (7 de mayo de 2019). La Nación. Obtenido de https://www.lanacion.com.ar/seguridad/ el-gobierno-oficializo-uso-armas-taser-parte-nid2245201.

El Gobierno volvió a calificar de "extorsivos" los cortes de calle de las organizaciones sociales pero llamó al diálogo para "canalizar diferencias". (21 de septiembre de 2019). Infobae. Obtenido de https://www.infobae.com/politica/2019/09/21/ el-gobierno-volvio-a-calificar-de-extorsivos-los-cortes-de-calle-de-lasorganizaciones-sociales-pero-llamo-al-dialogo-para-canalizar-diferencias/. 
Austral Comunicación

Volumen 9, número 2 (Diciembre de 2020): 553-582. ISSN 2313-9129

Fahsbender, F. (17 de abril de 2019). “Te voy a partir el alma si no retirás la denuncia”: los audios que recibió la menor violada por una manada en Florencio Varela. Infobae. Obtenido de https://www.infobae.com/sociedad/policiales/2019/04/17/ te-voy-a-partir-el-alma-si-no-retiras-la-denuncia-los-audios-que-recibio-lamenor-violada-por-una-manada-en-florencio-varela/.

Femicidio en Misiones: hallaron degollada a una joven y vecinos filmaron a un hombre cuando salía del lugar del crimen. (5 de abril de 2019). Infobae. Obtenido de https:// www.infobae.com/sociedad/policiales/2019/04/05/femicidio-en-misioneshallaron-degollada-a-una-joven-y-vecinos-filmaron-a-un-hombre-cuando-saliadel-lugar-del-crimen/.

Fernández Pedemonte, D. (2010). Conmoción pública. Los casos mediáticos y sus públicos ( $1^{a}$ ed.). Buenos Aires: La Crujía.

Ferree, M. M., Gamson, W. A., Gerhards, J. y Rucht, D. (2002). Shaping Abortion Discourse. Democracy and the Public Sphere in Germany and the United States. Cambridge: Cambridge University Press.

Galar, S. y Focás, B. (2019). El regreso de las víctimas. Reconfiguraciones en el procesamiento público de la inseguridad en la actual coyuntura política nacional (2016-2017). Austral Comunicación, 8(1), 131-150. Obtenido de https://doi. org/10.26422/aucom.2019.0801.gal.

Gallo, D. (2019, 26 de abril). Implementarán medidas policiales inmediatas para frenar la violencia de género. La Nación. Obtenido de https://www.lanacion.com. ar/seguridad/implementaran-medidas-policiales-inmediatas-frenar-violenciagenero-nid2242107.

García-Alonso Montoya, P. (2014). La empresa informativa busca nuevos modelos de negocio. Historia y Comunicación Social, 19, 729-741. Obtenido de https://doi. org/10.5209/rev_HICS.2014.v19.45173.

Gil, A. S., Zunino, E., Marín, J., Hasan, V. F., Pizarro, T., Pessolano, D., Di Paolo, B., Ilardo, I. J., López, J. y Fiochetta, M. (2019). Medios, género y delito: tramas y sentidos sobre violencia contra las mujeres. Cuestiones de género: de la igualdad y la diferencia, 14, 29-50. Obtenido de https://doi.org/10.18002/cg.v0i14.5834.

Iyengar, S. (1990). The Accessibility Bias in Politics: Television News and Public Opinion. Public opinion research, 2(1), 1-15.

Kessler, G. (2009). El sentimiento de inseguridad. Sociología del temor al delito (1 $1^{\mathrm{a}}$ ed.). Buenos Aires: Siglo XXI.

Kessler, G. (2014). Controversias sobre la desigualdad. Argentina, 2003-2013 (1ª ed.). Buenos Aires: Fondo de Cultura Económica.

Kessler, G. y Focas, B. (2014). ¿Responsables del temor? Medios y sentimiento de inseguridad en América Latina. Nueva Sociedad, 249, 137-148. 
Esteban Zunino

Vivir con miedo: un estudio sobre las agendas digitales y las fuentes de información del delito, la violencia y el riesgo

Koziner, N. (2018a). Periodistas y fuentes en la prensa argentina. Revisión teórica a partir de un caso empírico. Revista Mexicana de Opinión Pública, 24, 147-167. Obtenido de https://doi.org/10.22201/fcpys.24484911e.2018.24.61707.

Koziner, N. (2018b). Standing de las fuentes periodísticas en la política de medios argentina ( 2009 y 2016). Cuadernos Info, 42, 141-158.

Krippendorff, K. (1990). Metodología de análisis de contenido. Teoría y práctica (1ª ed.). Barcelona: Paidós.

Lagos, M. y Dammert, L. (2012). Latinobarómetro: La Seguridad Ciudadana El problema principal de América Latina. Obtenido de http://www.latinobarometro. org/documentos/LATBD_La_seguridad_ciudadana.pdf.

Las 19 propiedades del secretario de Néstor Kirchner que serán rematadas de forma "inmediata". (21 de junio de 2019). Infobae. Obtenido de https://www.infobae.com/ politica/2019/06/21/las-19-propiedades-del-secretario-de-nestor-kirchner-queseran-rematadas-de-forma-inmediata/.

Martini, S. (2007). Argentina, prensa gráfica, delito e inseguridad. En Rey, G. (ed.). Los relatos periodísticos del crimen ( $1^{\mathrm{a}}$ ed., pp. 21-54). Bogotá: Friedrich-Ebert-Stiftung/ Centro de Competencia en Comunicación para América Latina.

Martini, S. (2012). Opinión pública, medios masivos y control social. ¿Los bárbaros están entre nosotros? En Martini, S. y Contursi, M. E. (eds.).Comunicación pública del crimen y gestión del control social ( $1^{a}$ ed., pp. 19-46). Buenos Aires: La Crujía.

Martini, S. (2017). Últimas noticias. Construyendo la actualidad en el siglo XXI. En Martini, S. y Pereyra, M.(eds.). La noticia hoy. Tensiones entre la política, el mercado y la tecnología (1 $1^{a}$ ed., pp. 1-28). Buenos Aires: Imago Mundi.

Martini, S. y Luchessi, L. (2004). Los que hacen la noticia: periodismo, información y poder ( $1^{\text {a }}$ ed.). Buenos Aires: Biblos.

McCombs, M. (2006). Estableciendo la agenda. El impacto de los medios en la opinión pública y el conocimiento ( $1^{\mathrm{a}}$ ed.). Barcelona: Paidós Ibérica.

McCombs, M. y Valenzuela, S. (2014). Agenda-Setting Theory: The Frontier Research Questions (Número Sep 2014). Obtenido de https://doi.org/10.1093/ oxfordhb/9780199793471.013.48.

Meraz, S. (2011). Using Time Series Analysis to Measure Intermedia Agenda-Setting Influence in Traditional Media and Political Blog Networks. Journalism y Mass Communication Quarterly, 88(1), 176-194.

Messi, V. (11 de abril de 2019). Investigan la huella de Los Cuinis, los narcos mexicanos que se instalaron en Argentina. Clarín. Obtenido de https://www.clarin.com/ policiales/investigan-huella-cuinis-narcos-mexicanos-instalaron-argentina_0_ NsP5QPX1x.html.

Mitchelstein, E. y Boczkowski, P. J. (2017). Juventud, estatus y conexiones. 
Austral Comunicación

Volumen 9, número 2 (Diciembre de 2020): 553-582. ISSN 2313-9129

Explicación del consumo incidental de noticias en redes sociales. Revista Mexicana de Opinión Pública, 24, 131-145. Obtenido de https://doi.org/10.22201/ fcpys.24484911e.2018.24.61647.

Neuendorf, K. (2002). The content analysis guidebook ( $1^{\mathrm{a}}$ ed.). Thousand Oaks: Sage.

Otro trágico accidente en Carmen de Areco: ocho personas murieron tras un choque entre dos autos. (14 de abril de 2019). Infobae. Obtenido de https://www.infobae. com/sociedad/2019/04/14/otro-tragico-accidente-en-carmen-de-areco-ochopersonas-murieron-tras-un-choque-entre-dos-autos/.

Patricia Bullrich y el paro: "Generan caos, miedo y riesgo país, con episodios cuasi terroristas". (29 de abril de 2019). Clarín. Obtenido de https://www.clarin.com/ politica/patricia-bullrich-paro-30-abril-generan-caos-miedo-riesgo-pais_0_ iNn3IDNY8.html.

Pellegrini, S. y Mujica, M. C. (2006). Valor Agregado Periodístico (VAP): la calidad periodística como un factor productivo en un entorno medial complejo. Palabra Clave, 9(1), 7-18.

Pellegrini, S., Puente, S., Porath, W., Mujica, C. y Grassau, D. (2011). Valor agregado periodístico. La apuesta por la calidad de las noticias ( $1^{\mathrm{a}} \mathrm{ed}$.). Santiago de Chile: Pontificia Universidad Católica de Chile.

Pride, R. A. (1995). How Activists and Media Frame Social Problems: Critical Events Versus Performance Trends for Schools. Political Communication, 12(1), 5-26.

Retegui, L. (2017). Los procesos de organización del trabajo en la redacción de un diario. Un estudio a partir del diario La Nación, en el contexto digital (1995-2013). Buenos Aires: Universidad Nacional de Quilmes.

Rodríguez Alzueta, E. (2014). Temor y control: la gestión de la inseguridad como forma de gobierno ( $1^{\mathrm{a}} \mathrm{ed}$.). Buenos Aires: Futuro Anterior Ediciones.

Rost, A. y Bergero, F. (2016). Argentina. En Salaverría, R. (ed.). Ciberperiodismo en Iberoamérica (1 ${ }^{a}$ ed., pp. 1-19). Barcelona: Ariel.

Ruiz, I. (27 de mayo de 2019). El patrimonio de Alberto Fernández, bajo la lupa: vive en un departamento de Pepe Albistur. Obtenido de https://www.lanacion.com.ar/ politica/alberto-nid2252030.

Salaverría, R. (Ed.). (2016). Ciberperiodismo en Iberoamérica (1ª ed.). Barcelona: Ariel.

Sánchez Sandoval, A. (2013). Seguridad Nacional y Derechos Humanos. México, D. F.: Universidad Autónoma de México.

Salinas, L. (21 de agosto de 2019). Anulan un fallo por el Soterramiento del tren Sarmiento y favorecen a exfuncionarios K. Clarín. Obtenido de https://www.clarin.com/politica/ anulan-fallo-soterramiento-favorecen-ex-funcionarios-k_0_M5ColB0k-.html.

Scolari, C. A. (2014). Narrativas transmedia: nuevas formas de comunicar en la era digital. Anuario AC/E de cultura digital, 2014, 71-81. 
Sistema de Información Cultural de la Argentina. (2017). Encuesta Nacional de Consumos Culturales 2017. Ministerio de Cultura. Obtenido de https://www.sinca. gob.ar/Encuestas.aspx.

Sozzo, M. (2016). Postneoliberalismo y penalidad en América del Sur. A modo de introducción. En Sozzo, M. (ed.). Postneoliberalismo y penalidad en América del

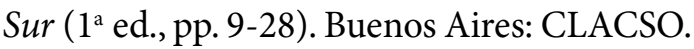

Steele,J.(1997). Don't Ask, Don't Tell, Don't Explain: Unofficial Sources and Television Coverage of the Dispute Gays in the Military. Political Communication, 14, 83-96. Obtenido de https://doi.org/https://doi.org/10.1080/105846097199551.

Téramo, M. T. (2006). Calidad de la información periodística en Argentina. Estudio de diarios y noticieros. Palabra Clave, 9(1), 57-84.

Tras los disturbios, los piqueteros amenazan con acampar en Plaza de Mayo. (11 de septiembre de 2019). La Nación. Obtenido de https://www.lanacion.com.ar/ politica/el-polo-obrero-confirmo-acampe-48-horas-nid2286765.

Vaca, P. (29 de septiembre de 2019). Calentamiento global: parece que esta vez el lobo viene de verdad. Clarín. Obtenido de https://www.clarin.com/opinion/ calentamiento-global-parece-vez-lobo-viene-verdad_0_2QgLOWpb.html.

Vilker, S. (2006). Truculencia. La prensa policial entre el terrorismo de estado y la inseguridad ( $1^{\mathrm{a}}$ ed.). Buenos Aires: Licenciatura en Ciencias de la Comunicación, Facultad de Ciencias Sociales, Universidad de Buenos Aires/Prometeo.

Zucker, H. G. (1978). The Variable Nature of News Media Influence. En Rubin, B. D. (ed.). Communication Yearbook 2 (pp. 225-245). New Brunswick: Transaction Publishers. 